TITLE:

\title{
Direct evidence of the surface track potential
}

$\operatorname{AUTHOR}(\mathrm{S})$ :

Nakajima, Kaoru; Sakata, Masakazu; Suzuki, Motofumi; Kimura, Kenji

CITATION:

Nakajima, Kaoru ... [et al]. Direct evidence of the surface track potential. PHYSICAL REVIEW A 2010, 82(2): 022901.

\section{ISSUE DATE:}

2010

URL:

http://hdl.handle.net/2433/148385

RIGHT:

(C) 2010 The American Physical Society 
PHYSICAL REVIEW A 82, 022901 (2010)

\title{
Direct evidence of the surface track potential
}

\author{
Kaoru Nakajima, Masakazu Sakata, Motofumi Suzuki, and Kenji Kimura* \\ Department of Micro Engineering, Kyoto University, Yoshida-honmachi, Sakyo, Kyoto 606-8501, Japan
}

(Received 22 May 2010; published 3 August 2010)

\begin{abstract}
Angular and energy distributions of fragment protons dissociated from $\mathrm{HeH}^{+}$during grazing-angle scattering from a $\mathrm{KCl}(001)$ surface are measured. The surface of $\mathrm{KCl}(001)$ is heated at $180^{\circ} \mathrm{C}$ and the beam current is kept lower than $1 \mathrm{fA}$ to prevent macroscopic surface charging. The angular distribution of the fragment protons shows a well-defined peak similarly to the grazing-angle scattering of atomic ions. The observed peak, however, is shifted from the specular angle toward larger scattering angles. The observed angular shift for the trailing proton is larger than that for the leading proton. These results clearly indicate that the motion of the fragment protons is affected by the surface track potential induced by the partner He ion.
\end{abstract}

DOI: 10.1103/PhysRevA.82.022901

PACS number(s): 79.20.Rf, 79.60.Bm

\section{INTRODUCTION}

When an energetic ion passes through solids, the projectile ion ionizes the target atoms and a number of vacancies (holes) are created along the ion trajectory. This results in a positive nuclear track potential behind the ion. Although conduction electrons promptly fill the vacancies in conductive materials, the filling process is much slower than the ionsolid interaction time in the case of insulators. Thus, the induced track potential may affect ion-solid interactions, such as secondary electron emission, energy loss, and charge exchange.

The first evidence of the track potential was claimed through the measurement of Auger electrons [1]. Energy shift of carbon KLL Auger electrons up to $68 \mathrm{eV}$ was observed when $100 \mathrm{MeV} \mathrm{Ne}^{9+}$ ions were passing through thin polypropylene foils. The observed energy shift was ascribed to the effect of the nuclear track potential. Similar energy shift was also observed for convoy electrons emitted by $5 \mathrm{MeV} / \mathrm{u}$ heavy ions passing through polypropylene foils [2], although the observed energy shift is less than $1 \%$ of the convoy electron energy. A more pronounced effect was observed by Gómez et al. using grazing-angle scattering of $60-100 \mathrm{keV} \mathrm{H}^{+}$ions at $\mathrm{LiF}$ (001) surfaces [3]. They observed that the convoy electrons are decelerated by $5-13 \mathrm{eV}$, which is as large as $15 \%-25 \%$ of the convoy electron energy, indicating that a strong surface track potential is induced during the grazing-angle scattering of fast ions at insulator surfaces. In a new measurement with a smooth LiF (001) surface, however, they observed acceleration of convoy electrons by $5 \mathrm{eV}$ [4] instead of deceleration. This acceleration was semiquantitatively explained in terms of the surface wake potential (dynamical image potential), suggesting that the effect of the surface track potential is negligibly small. Thus, the existence of the surface track potential is now a subject of controversy.

The difficulty in this kind of measurements is influences of macroscopic charging. It is believed that if the insulators like alkali-metal halides are heated at proper temperatures $\left(>100^{\circ} \mathrm{C}\right)$, the macroscopic charging can be avoided by the ionic conduction. This is true when the beam current is far below $1 \mathrm{pA}$ [5]. In order to measure the energy spectrum

\footnotetext{
*kimura@kues.kyoto-u.ac.jp
}

of the convoy electrons with good statistics, however, a relatively high beam current is required because the production probability of the convoy electron is usually very small. Such a high beam current induces the macroscopic charging even if the insulator surface is heated [5]. Other probing methods compatible with low beam current far below $1 \mathrm{pA}$ are absolutely necessary for studying the surface track potential in detail.

In the present article, the surface track potential is studied using a $\mathrm{HeH}^{+}$ion as a probe. The angular and energy distributions of fragment protons dissociated from $\mathrm{HeH}^{+}$ during grazing-angle scattering at a $\mathrm{KCl}(001)$ surface is observed. The motion of the protons should be affected by the surface track potential induced by the partner $\mathrm{He}$ ion as well as the proton itself. Because almost all incident ions are reflected from the surface in a narrow range of angles centered at the specular angle, the angular and energy distributions can be measured with good statistics even if the beam current is reduced below $1 \mathrm{fA}$. Thus, the effect of the surface track potential on the distributions can be observed in detail without suffering from the macroscopic charging.

\section{EXPERTIMENTAL}

A beam of $1 \mathrm{MeV} \mathrm{HeH}^{+}$ions was produced by the 4-MV Van de Graaff accelerator of Kyoto University. The beam was collimated by a series of four-jaw slit systems to less than $0.1 \times 0.1 \mathrm{~mm}^{2}$ in size and less than $0.5 \mathrm{mrad}$ in divergence. The collimated beam was incident on the surface of the target crystal at grazing angles. The target crystal was mounted on a five-axis high-precession goniometer in an ultrahigh-vacuum (UHV) scattering chamber (base pressure $3 \times 10^{-8} \mathrm{~Pa}$ ). The energy spectra of the scattered ions were measured by a $90^{\circ}$ sector magnetic spectrometer (energy resolution $<0.1 \%$ ). The spectrometer had a small aperture (diameter $1.5 \mathrm{~mm}$ ) at the entrance focal point and a one-dimensional position sensitive detector (1D-PSD) at the exit focal plane. The distance between the target and the entrance aperture was $680 \mathrm{~mm}$ (the acceptance angle of the entrance aperture is $\pm 1.1 \mathrm{mrad}$ ). Two sets of parallel plate electrostatic deflectors were installed between the target and the entrance aperture so that ions scattered at any scattering and azimuth angles 
$(\theta$ and $\varphi$ ) can pass through the aperture by applying appropriate voltages on these deflectors. This allows precise energy measurements of the ions scattered at any direction within $\pm 2^{\circ}$ from the incident direction without moving the heavy spectrometer.

Single crystals of $\mathrm{KCl}(001)$ and SnTe (001) were used as targets. While both crystals have a NaCl-type structure and the lattice constants are almost the same, the electronic structures are very different; that is, $\mathrm{KCl}$ is a typical insulator but $\mathrm{SnTe}$ is a narrow gap semiconductor. The effect of the surface track potential was studied with $\mathrm{KCl}(001)$ and $\mathrm{SnTe}$ (001) was used as a reference material. A single crystal of $\mathrm{KCl}$ was cleaved along the (001) plane in air and was mounted on the goniometer. After evacuation and baking the UHV chamber at $150^{\circ} \mathrm{C}$ for $24 \mathrm{~h}$ the $\mathrm{KCl}(001)$ was heated at $270^{\circ} \mathrm{C}$ for $1 \mathrm{~h}$ to prepare a clean surface. The surface thus prepared was atomically flat and the mean step density observed by atomic force microscope was about $5 \times 10^{-3} \mathrm{~nm}^{-1}$ [6]. During the measurement, the surface was kept at $180^{\circ} \mathrm{C}$ to prevent macroscopic surface charging. A single crystal of SnTe (001) was prepared in situ by epitaxial growth on the $\mathrm{KCl}(001)$ at $240^{\circ} \mathrm{C}$ under UHV conditions. High-purity SnTe $(99.999 \%)$ was evaporated at a low growth rate (less than $0.5 \mathrm{~nm} / \mathrm{min}$ ) for several hours to prepare an atomically flat surface.

\section{RESULTS AND DISCUSSION}

Figure 1 shows the examples of the observed energy spectra of the fragment protons when $1 \mathrm{MeV} \mathrm{HeH}^{+}$ions were incident on $\mathrm{KCl}$ (001) and SnTe (001). Due to the Coulomb explosion, the energy spectra have two peaks. The high energy peak at $\sim 200 \mathrm{keV}$ corresponds to the leading protons and the low energy peak at $\sim 193 \mathrm{keV}$ corresponds to the trailing protons. The observed peak separation is about $6.5 \mathrm{keV}$ for both $\mathrm{KCl}$ (001) and SnTe (001). The release energy during the Coulomb explosion is estimated to be $\sim 17 \mathrm{eV}$, which is in rough agreement with the release

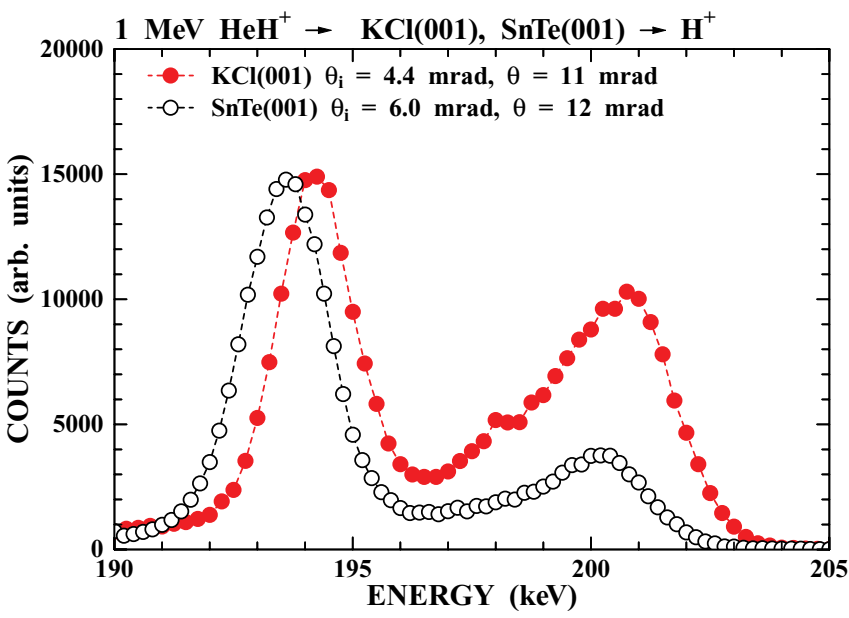

FIG. 1. (Color online) An example of the energy spectrum of the fragment protons observed at $\theta=11 \mathrm{mrad}$ when $1 \mathrm{MeV} \mathrm{HeH}^{+}$ ions were incident on $\mathrm{KCl}(001)$ at $\theta_{i}=4.4 \mathrm{mrad}$. The result for $\mathrm{SnTe}(001)$ observed at $\theta=12 \mathrm{mrad}$ and $\theta_{i}=6 \mathrm{mrad}$ is also shown for comparison. (a)

(b)

(c)

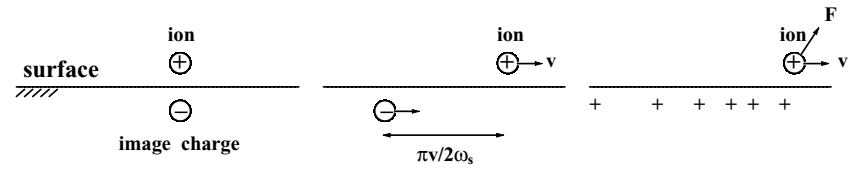

FIG. 2. (a) Image charge induced by a positive ion at surface. (b) Dynamical image charge induced by a positive ion traveling parallel to the surface plane. (c) Holes crated by He ion at insulator surface, which are the origin of the surface track potential.

energy for the dissociation into $\mathrm{He}^{+}+\mathrm{H}^{+}$. This indicates that the $\mathrm{He}^{+}$fraction is dominant during the Coulomb explosion.

In the case of SnTe (001), the trailing peak is much larger than the leading peak, similar to the foil transmission experiments [7]. This can be understood by the dynamical image charge (surface wake) induced by the partner He ion. When a positive ion approaches a surface, a negative image charge is induced [see Fig. 2(a)]. If the ion moves parallel to the surface, the induced image charge follows the ion a distance $\pi v / 2 \omega_{s}(\sim 0.7 \mathrm{~nm}$ in the present case) behind the ion, where $\omega_{s}$ is the frequency of the surface plasmon [see Fig. 2(b)]. The dynamical image charge induced by the partner He ion pulls the proton backward during the Coulomb explosion of $\mathrm{HeH}^{+}$and this results in enhancement of the trailing peak [8]. On the other hand, in the case of $\mathrm{KCl}(001)$, the surface track potential induced by the partner $\mathrm{He}$ ion pushes the proton forward as is schematically shown in Fig. 2(c). Consequently, the yield of the leading proton is expected to be enhanced. The observed result, however, shows that the yield of the leading peak is almost the same as that of the trailing peak. This suggests that neither the surface track potential nor the dynamical image is induced on $\mathrm{KCl}(001)$. Even in an insulator surface, however, the dynamical image should be induced, although it is reduced by $(\varepsilon-1) /(\varepsilon+1)$, where $\varepsilon$ is dielectric constant of the insulator $(\varepsilon=4.85$ for $\mathrm{KCl}$ ). Actually, image acceleration of multiply charged ions was observed at a $\mathrm{LiF}$ (001) surface [9]. Thus, the present result strongly suggests the existence of the surface track potential, which accidentally cancels the effect of the dynamical image on the motion of the fragment proton. Although this might be circumstantial evidence of the surface track potential, direct evidence could be provided by measuring the angular distributions of fragment protons.

Figure 3 shows the scattering angle distribution of the fragment protons observed at $\varphi=0 \mathrm{mrad}$ when $1 \mathrm{MeV} \mathrm{HeH}^{+}$ ions are incident on $\mathrm{KCl}(001)$ at a grazing angle of incidence, $\theta_{i}$, of $4.4 \mathrm{mrad}$. The distribution has a well-defined peak like the grazing-angle scattering of atomic ions but the peak position $(\theta=11 \mathrm{mrad})$ is shifted from the specular angle $(\theta=$ $8.8 \mathrm{mrad})$ toward larger angle by $2.2 \mathrm{mrad}$. Such deviation was not observed for SnTe (001). The observed deviation can be explained in terms of the surface track potential. The surface track potential pushes the positive ion outward from the surface [Fig. 2(c)]. In the incoming (outgoing) trajectory, the surface track potential decelerates (accelerates) the perpendicular motion of the projectile ion. For atomic ions the deceleration and the acceleration cancel each other and the ions are scattered at the specular angle. In the case of $\mathrm{HeH}^{+}$, the 


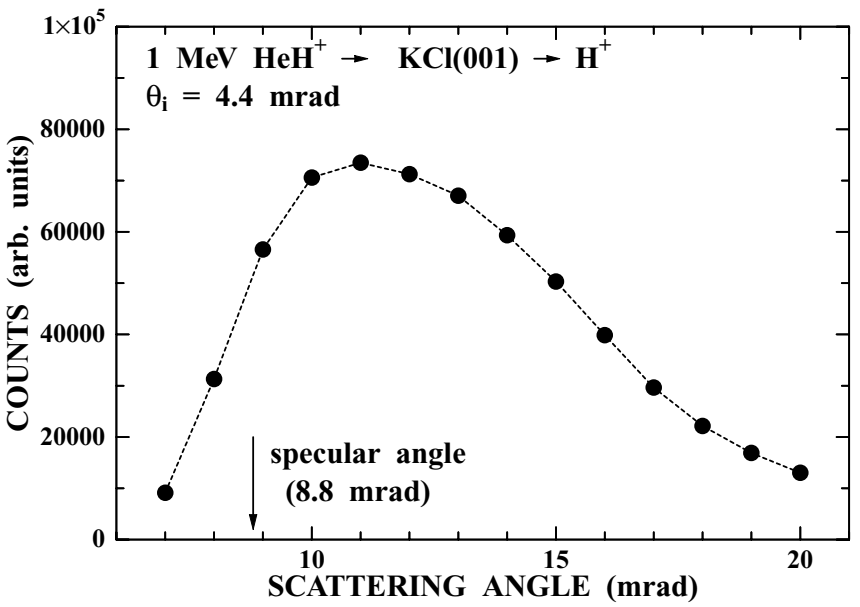

FIG. 3. Scattering angle distributions of protons and He ions observed at $\varphi=0 \mathrm{mrad}$ when $1 \mathrm{MeV} \mathrm{HeH}^{+}$ions are incident on $\mathrm{KCl}(001)$ at $\theta_{i}=4.4 \mathrm{mrad}$.

dissociation occurs around the apex of the trajectory, that is, during the closest approach to the surface plane. The acceleration of the fragment proton after dissociation is larger than the deceleration of $\mathrm{HeH}^{+}$before the dissociation because the acceleration (deceleration) is inversely proportional to the projectile mass. Consequently, the fragment protons are reflected at scattering angles larger than the specular angle. Thus, the observed shift of the scattering angle seems to be evidence of the surface track potential. The macroscopic charging, however, causes similar angular shift. In order to verify that the observed angular shift is caused by the surface track potential, the induced surface track potential was estimated using a simple model proposed in our previous article [10].

The hole production rate when the fragment He ion travels at $z$ from the surface can be calculated with a binary encounter model,

$$
P_{h}(z)=\frac{2 \pi Z_{\mathrm{eff}}^{2} e^{4}}{m v^{2}} \sum_{i} n_{i}(z)\left(\frac{1}{\varepsilon_{i}}-\frac{1}{2 m v^{2}}\right),
$$

where $Z_{\text {eff }}$ is the effective charge $\left(Z_{\text {eff }}^{2}\right.$ was determined to be 3.3 from the observed charge state distribution of the fragment $\mathrm{He}$ ions), $m$ is the electron mass, $n_{i}(z)$ is the electron density of the $i$ th shell averaged over the plane parallel to the surface, and $\varepsilon_{i}$ is its binding energy. We employed Hartree-Fock wave functions of isolated $\mathrm{K}$ and $\mathrm{Cl}$ atoms to calculate $n_{i}(z)$ [11]. When the holes are created they are screened by the ejected electrons. The screening becomes weaker as these electrons move away from the surface. A simple screening function $\Phi(t, z)=1-$ $\exp \left(-V_{\mathrm{SE}} t / z\right)$ was introduced in our previous article, where $V_{\mathrm{SE}}$ is the velocity of the secondary electrons and $t$ is the time after the creation of the hole. Using this simple model with $V_{\mathrm{SE}}=0.5$ a.u., the observed effect of the surface track potential on the stopping power of atomic projectile ions was successfully reproduced [10]. We use this model to calculate the surface track potential induced by the fragment He ion and the force acting on the partner proton was estimated for various positions of the proton with respect to the He ion. Examples of the perpendicular component, $F_{z}$, of the calculated force

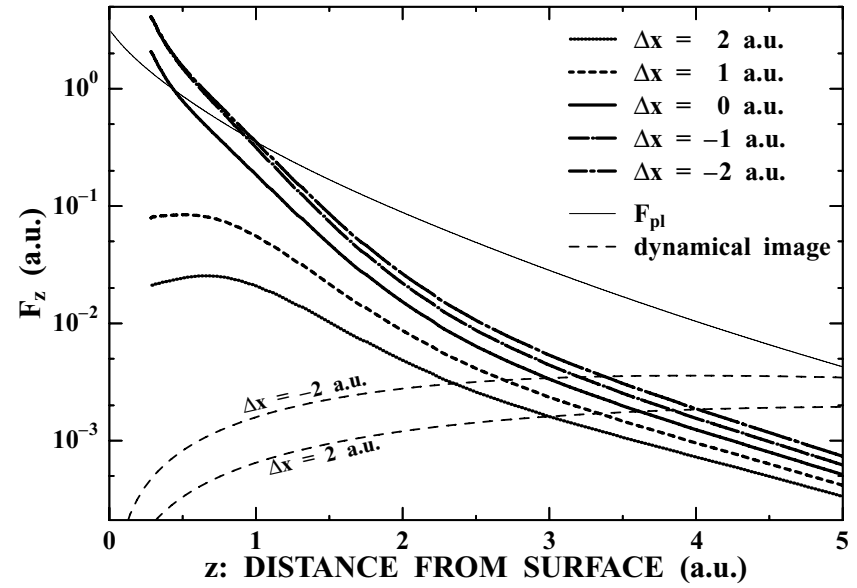

FIG. 4. Perpendicular component of the force acting on the fragment proton by the surface track potential induced by the partner He ion. Shown are the results for $\Delta x=0, \pm 1$, and \pm 2 a.u. (where positive values correspond to the leading proton). For comparison, the force by the surface continuum potential is shown by a thin solid line. The perpendicular force due to the dynamical image charge induced by the partner He ion for $\Delta x= \pm 2$ a.u. is also shown by dashed lines.

when the He-H axis is aligned to the velocity direction are shown in Fig. 4. Shown are the results for $\Delta x=0, \pm 1$, and \pm 2 a.u., where $\Delta x$ is the internuclear distance between the $\mathrm{He}$ ion and the proton (positive values correspond to the leading proton). For comparison, the force, $F_{\mathrm{pl}}$, due to the surface continuum potential is also shown. The calculated $F_{z}$ for the leading proton is one order of magnitude smaller than $F_{\mathrm{pl}}$. For the trailing proton, $F_{z}$ increases very rapidly with decreasing $x$ and becomes comparable or even larger than $F_{\mathrm{pl}}$ at $z \leqslant 0.5 \AA$. Considering that the distance of the closest approach to the surface is $0.45 \AA$ for a $0.8-\mathrm{MeV} \mathrm{He}$ ion at $\theta_{i},=4.4 \mathrm{mrad}$, the trailing protons should be significantly affected by the surface track potential, while the leading protons are not. This suggests that the observed angular shift is mainly responsible to the trailing protons.

Figure 5 shows examples of the observed joint distributions in the $E-\theta$ plane at $\varphi=0 \mathrm{mrad}$ for $\mathrm{KCl}$ (001) [Fig. 5(a)] and SnTe (001) [Fig. 5(b)]. Unlike the foil transmission experiments, the fragment protons do not distribute on an ellipse. Instead, the protons form two peaks corresponding to the leading and trailing protons. This indicates that the molecular axis tends to align to the surface during the grazingangle scattering. This alignment is caused by the continuum surface potential acting on the fragment ions at the surface [12]. If the molecular axis inclines to the surface, the constituent atom which is closer to the surface feels stronger repulsive force from the surface. Consequently, the molecular axis tends to align to the surface. Although both distributions show this alignment effect, there is a pronounced difference between $\mathrm{KCl}$ (001) and SnTe (001). While both peaks are located at the specular angle $(\theta=12 \mathrm{mrad})$ for $\operatorname{SnTe}(001)$, the peak positions are shifted from the specular angle $(\theta=8.8 \mathrm{mrad})$ toward larger $\theta$ for $\mathrm{KCl}(001)$, as is shown in Fig. 3. The angular shift of the trailing protons (low energy peak) is about 3 mrad while the leading protons (high energy peak) show only very small shift ( $\sim 0.5 \mathrm{mrad})$. As was discussed earlier, such difference between the leading and trailing protons can 
(a) $1 \mathrm{MeV} \mathrm{HeH}^{+} \rightarrow \mathrm{KCl}(001), \theta_{\mathrm{i}}=4.4 \mathrm{mrad}$

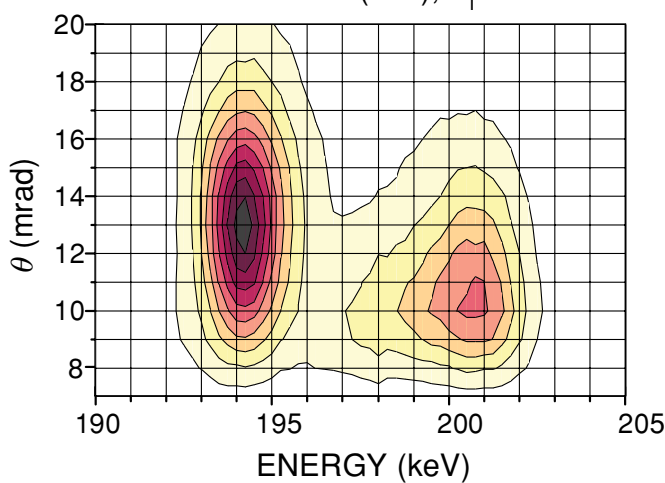

(b)

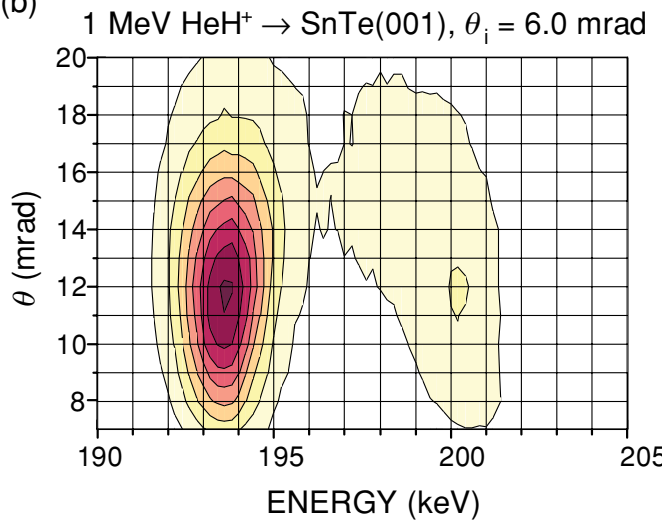

FIG. 5. (Color online) Joint distributions in the $E-\theta$ plane for fragment protons measured at $\varphi=0 \mathrm{mrad}$ when $1 \mathrm{MeV} \mathrm{HeH}^{+}$ions were incident on $\mathrm{KCl}(001)$ at $\theta_{i}=4.4 \mathrm{mrad}$ (a) and on $\mathrm{SnTe}(001)$ at $\theta_{i}=6 \mathrm{mrad}(\mathrm{b})$.

be explained by the surface track potential but not by the macroscopic charging, confirming that the motion of the fragment proton is affected by the surface track potential induced by the partner He ion.

Finally, we discuss the effect of the dynamical image charge on the scattering angle. The fragment $\mathrm{He}$ ion induces dynamical image charge, which may also affect the perpendicular motion of the partner proton. The perpendicular force acting on the trailing and leading protons due to the dynamical image charge can be given by

$$
F_{z}^{\mathrm{img}} \approx-\frac{\varepsilon-1}{\varepsilon+1} \frac{Z_{\mathrm{eff}} e^{2} 2 z}{\left[\left(\pi v / 2 \omega_{s} \pm \Delta x\right)^{2}+4 z^{2}\right]^{3 / 2}} .
$$

The calculated $\left|F_{z}^{\mathrm{img}}\right|$ with $\Delta x=2$ a.u. is shown by dashed lines in Fig. 4, which is much smaller than $F_{z}$ around the closest approach to the surface plane. Thus, the effect of the dynamical image charge on the scattering angle should be much smaller than that of the surface track potential.

\section{CONCLUSION}

The surface track potential has been studied using $\mathrm{HeH}^{+}$ ions as a probe. The angular and energy distributions of the fragment protons were measured when $1 \mathrm{MeV} \mathrm{HeH}+$ ions were scattered from $\mathrm{KCl}(001)$ under grazing incidence. The surface of $\mathrm{KCl}(001)$ was heated at $180^{\circ} \mathrm{C}$ and the beam current was kept lower than $1 \mathrm{fA}$ to prevent macroscopic surface charging. The observed angular distribution of the fragment protons has a peak at a scattering angle slightly larger than the specular angle. The observed angular shift can be explained by either the surface track potential or the macroscopic charging. By measuring the joint distribution of the fragment protons in the $E-\theta$ plane, it is found that the angular shift of the trailing protons is larger than that of the leading protons. This clearly indicates that the observed angular shift is caused by the surface track potential.

\section{ACKNOWLEDGMENTS}

We would like to thank the members of the Quantum Science and Engineering Center of Kyoto University for the use of the Van de Graaff accelerator. We are grateful to Messrs. $\mathrm{K}$. Yoshida and K. Norisawa for their assistance in operating the accelerator.
[1] G. Schiwietz et al., Phys. Rev. Lett. 69, 628 (1992).

[2] G. Xiao, G. Schiwietz, P. L. Grande, N. Stolterfoht, A. Schmoldt, M. Grether, R. Köhrbrück, A. Spieler, and U. Stettner, Phys. Rev. Lett. 79, 1821 (1997).

[3] G. R. Gómez, O. Grizzi, E. A. Sánchez, and V. H. Ponce, Phys. Rev. B 58, 7403 (1998).

[4] V. H. Ponce, L. F. de Ferrariis, O. Grizzi, M. L. Martiarena, and E. A. Sánchez, Nucl. Instrum. Methods B 232, 37 (2005).

[5] K. Nakajima, A. Nakamoto, M. Suzuki, and K. Kimura, Nucl. Instrum. Methods B 248, 21 (2006).

[6] K. Narumi, Y. Fujii, K. Kimura, M. Mannami, and H. Hara, Surf. Sci. 303, 187 (1994).
[7] D. S. Gemmell, J. Remillieux, J.-C. Poizat, M. J. Gaillard, R. E. Holland, and Z. Vager, Phys. Rev. Lett. 34, 1420 (1975).

[8] Y. Susuki, T. Ito, K. Kimura, and M. Mannami, J. Phys. Soc. Jpn. 61, 3535 (1992).

[9] C. Auth, T. Hecht, T. Igel, and H. Winter, Phys. Rev. Lett. 74, 5244 (1995).

[10] K. Kimura, S. Usui, T. Tsujioka, S. Tanaka, K. Nakajima, and M. Suzuki, Vacuum 73, 59 (2004).

[11] H. Herman and S. Skillman, Atomic Structure Calculations (Prentice-Hall, Englewood Cliffs, NJ, 1963).

[12] Y. Susuki, M. Mukai, K. Kimura, and M. Mannami, Nucl. Instrum. Methods B 48, 347 (1990). 